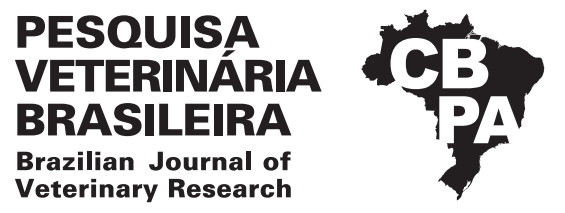

Pesq. Vet. Bras. 38(9):1720-1725, setembro 2018 DOI: 10.1590/1678-5150-PVB-5313

Artigo Original

Animais de Produção/Livestock Diseases

ISSN 0100-736X (Print)

ISSN 1678-5150 (Online)

\title{
Bem-estar em equinos do Jockey Club do Paraná: indicadores clínicos, etológicos e ritmo circadiano do cortisol $^{1}$
}

\author{
Lilian A. Gontijo ${ }^{2}$, Fabiane Cassou², Patrícia C. Duarte², Luiz A. Lago ${ }^{2}$, \\ Geraldo E.S. Alves ${ }^{2}$, Marília M. Melo² e Rafael R. Faleiros ${ }^{2 *}$
}

\begin{abstract}
Gontijo L.A., Cassou F., Duarte P.C., Lago L.A., Alves G.E.S., Melo M.M. \& Faleiros R.R. 2018. [Welfare in horses of the Jockey Club Paraná: clinic ethologic indicators and circadian rhythm of cortisol.] Bem-estar em equinos do Jockey Club do Paraná: indicadores clínicos, etológicos e ritmo circadiano do cortisol. Pesquisa Veterinária Brasileira 38(9):1720-1725. Escola de Veterinária, Universidade Federal de Minas Gerais, Av. Presidente Antônio Carlos 6627, Cx. Postal 567, Belo Horizonte, MG 31270-901, Brazil. E-mail: faleirosufmg@gmail.com

The objective was to study indicative parameters of welfare in 30 race horses evaluated for temperament, behavior, clinical parameters (general condition, abrasions, body score, colic in the last year, heart and respiratory rates) and plasma cortisol levels (morning, afternoon and circadian rhythm). All animals presented good general physical condition, normal clinical parameters for the species and no colic episodes in the year preceding the study. However, $36.7 \%$ of animals had stereotypies. Despite the high incidence of altered circadian cortisol rhythmicity (CCR) within the group of animals with abnormal behavior (63.64\%), there was no statistical difference $(\mathrm{P}>0.05)$ when compared with the incidence in the group animals without stereotyping (31.58\%). Four animals with stereotypic behavior carried devices to restrict the expression of anomalies. All these four individuals had altered CCR, showing that restricting the stereotypic behavior of a horse increases 18 times the chances of altered CCR when compared to an individual with normal behavior. The horses of the Jockey Club of Paraná, despite having good physical condition compared to previous national studies, had behavioral and adrenocortical function consonant with chronic stress changes. The results also corroborate previous concepts of stereotyping as stress relieving in horses, and that the use of its physical restriction increases the chances of disturbances in cortisol metabolism.
\end{abstract}

INDEX TERMS: Welfare, horses, Jockey Club Paraná, clinic indicator, ethologic indicator, circadian rhythm, cortisol, stabling, stress, thoroughbred.

RESUMO.- Com o objetivo de estudar parâmetros indicativos de bem-estar, 30 equinos de corrida foram avaliados quanto ao temperamento, comportamento, parâmetros clínicos (condição geral, escoriações, escore corporal, cólicas no último ano, frequências cardíaca e respiratória) e níveis de cortisol plasmático (matutino, vespertino e ritmo circadiano). Todos os animais apresentaram boas condições físicas gerais, parâmetros clínicos normais para a espécie e não tiveram cólica no ano anterior ao estudo, demonstrando a preocupação dos

\footnotetext{
${ }^{1}$ Recebido em 15 de agosto de 2017.

Aceito para publicação em 21 de dezembro de 2017.

${ }^{2}$ Escola de Veterinária, Universidade Federal de Minas Gerais (UFMG), Av. Presidente Antônio Carlos 6627, Cx. Postal 567, Belo Horizonte, MG 31270-901, Brasil.*Autor para correspondência: faleirosufmg@gmail.com
}

tratadores com a saúde física dos animais. Contudo, 36,7\% dos animais estudados apresentavam estereotipias. Apesar da alta incidência de indivíduos com ritmo circadiano do cortisol (RCC) alterado dentro do grupo de animais com comportamentos anormais $(63,64 \%)$, não foi encontrada diferença estatística $(\mathrm{P}>0,05)$ quando comparado com a incidência do grupo de animais sem estereotipia $(31,58 \%)$. Quatro animais com estereotipia portavam dispositivos para restringir a expressão de anomalias. Desses, todos apresentaram RCC alterado, permitindo demonstrar que equinos restringidos fisicamente de realizar sua estereotipia apresentam 18 vezes mais chances de ter alteração no RCC do que um animal de comportamento normal. Conclui-se que os equinos do Jockey Club do Paraná, apesar de apresentarem condições físicas satisfatórias comparados a estudos nacionais 
anteriores, possuíam alterações comportamentais e de função adrenocortical compatíveis com estresse crônico. Os achados também corroboram noções prévias de que a estereotipia é uma forma de tentar aliviar o estresse crônico em equinos, e que o uso de meios físicos para restringir sua manifestação aumenta a chance de distúrbios no metabolismo do cortisol.

TERMOS DE INDEXAÇÃO: Bem-estar, equinos, Jockey Club do Paraná, indicador clínico, indicador etológicos, ritmo circadiano, cortisol, estabulação, estresse, puro-sangue-inglês.

\section{INTRODUÇÃO}

A ciência do bem-estar animal e seus conceitos têm sido discutidos nas últimas décadas, especialmente em decorrência das condições impostas pelo homem aos animais domésticos e silvestres em cativeiro. Nesse contexto, essa ciência vem se desenvolvendo, cada vez mais, no sentido de criar formas objetivas e eficientes para se avaliar o bem-estar animal (Mason \& Latham 2004). Segundo Broom \& Molento (2004), tal avaliação está relacionada tanto a questões facilmente mensuráveis, como níveis hormonais e parâmetros fisiológicos, como a sentimentos inerentes ao indivíduo.

0 confinamento de cavalos em baias está associado ao desenvolvimento de estereotipias e outros comportamentos anormais, inclusive em animais que são submetidos a atividades físicas regulares, enquanto a privação de socialização com outros animais promove maior ocorrência de mordidas e coices nos treinadores, por exemplo (Søndergaard \& Ladewig 2004). Sabe-se que o estresse, agudo ou crônico, causa uma resposta neuroendócrina, a qual se relaciona intimamente com o comportamento. Os hormônios afetam o comportamento e o comportamento retroalimenta negativa ou positivamente os hormônios (Budzynska 2014). Diversos estudos têm utilizado os níveis de cortisol para avaliar os níveis de estresse de animais (Irvine \& Alexander 1994, Leal et al. 2011, Budzynska 2014, Ellis et al. 2014, Gontijo et al. 2014). Para tal é importante considerar as flutuações normais dos níveis de cortisol durante o dia. Os equinos, assim como outros mamíferos, apresentam ritmo circadiano de cortisol (RCC) com pico pela manhã e redução dos níveis sérios no período da tarde e noite (Irvine \& Alexander 1994). Essas flutuações são menores em animais submetidos ao estresse devido à ativação do sistema nervoso autônomo simpático e do eixo hipotálamo-hipofisário-adrenal, causando liberação do hormônio adrenocorticotrófico e estimulando a córtex da adrenal a secretar cortisol (Budzynska 2014, Gontijo et al. 2014). Segundo Douglas (1999), o RCC normal dos equinos é aquele em que a diferença das concentrações séricas de cortisol entre o período da manhã e da tarde (intervalo mínimo de 8 horas) possui diferença acima de 30\%. Considerando essa premissa, estudos têm mostrado relação entre alteração do RCC e outros indicadores de estresse crônico (Leal 2007, Leal et al. 2011, Gontijo et al. 2014).

Alguns autores sugeriram no passado que a observação de estereotipias (Barnett \& Hemsworth 1990) mesmo associadas à cortisolemia (Rushen 1991) não são confiáveis para interpretação do bem-estar animal. No entanto, a complexa interação entre a resposta individual a fatores estressantes, estereotipia e parâmetros fisiológicos foi extensamente estudada nos últimos anos (Mason \& Latham 2004), sendo que na espécie equina é recomendado o estudo da cortisolemia associado a alterações comportamentais (Hausberger et al. 2008).

No presente estudo examinou-se a hipótese de que equinos da raça Puro Sangue Inglês (PSI) estabulados no Jockey Club do Paraná, com idades entre dois e cinco anos, apresentam indicativos de estresse crônico como presença de estereotipias e alterações do ritmo circadiano de cortisol alterado. Adicionalmente, verificou-se a hipótese da existência de associação entre ocorrência de estereotipias e a ocorrência de RCC alterado nessa população.

O objetivo foi avaliar o bem-estar de equinos do Jockey Club do Paraná por meio da observação do temperamento e do comportamento dos animais, bem como da mensuração de parâmetros clínicos e do ritmo circadiano de cortisol. Mais além, a estudou-se uma possível associação de alterações de comportamento e com alterações de no ritmo circadiano de cortisol na população estudada.

\section{MATERIAL E MÉTODOS}

Foram utilizados 30 equinos da raça Puro Sangue Inglês (PSI) com idades entre dois e cinco anos estabulados no Jockey Club do Paraná (Hipódromo do Tarumã). A atividade física dos animais consistia de treinamento específico na raia de corrida pela manhã, com um dia de folga por semana, e passeio à tarde, com caminhada ao cabresto ou montados. No restante do tempo, os animais permaneciam confinados em baias individuais, recebendo quantidades adequadas de alimento e água de boa qualidade.

Dentro da avaliação clínica, classificou-se a condição corporal (adiposidade) utilizando-se uma escala de 1 a 9 , sendo 1 caquético e 9 o animal extremamente obeso, conforme previamente descrito (Carroll \& Huntington 1988). Logo após, realizou-se uma avaliação do estado geral do animal baseando-se na qualidade do pelame, crina e casco e presença de alterações de saúde evidentes, a fim de verificar se o animal estava bem cuidado ou não. 0 estado geral foi classificado em 5 graus, sendo o grau 1 péssimo, 2 ruim, 3 regular, 4 bom ou grau 5, ótimo. Posteriormente, foi feita avaliação quanto à presença de feridas e escoriações com classificação de 0 a 4 , sendo 0 ausente e o grau 4 com maior intensidade de lesões.

No exame físico, avaliou-se a frequência cardíaca com o auxílio de estetoscópio e a frequência respiratória pela realizada por contagem visual dos movimentos torácicos e auscultação com estetoscópio. Também como parte da avaliação de saúde dos equinos, os tratadores foram entrevistados quanto à ocorrência de cólicas nos últimos 12 meses.

Para se verificar a ocorrência de estereotipias, a observação de todos os animais foi feita na primeira semana de outubro, sempre pela mesma pesquisadora, e de forma individual. 0 período de observação de cada animal foi de 6 horas de um mesmo dia, sendo quatro horas durante o dia e duas horas durante a noite (Gontijo et al 2014). A avaliação foi realizada com os animais em seu próprio ambiente, mantendo uma distância entre a pesquisadora e o animal para que o mesmo não se interessasse pela presença humana. Foram considerados os tipos de comportamentos classificados como normais (distraído, alerta em estação, deitado, focinho rente ao chão e relinchar) ou anormais (agressividade, cavar, caminhada estereotípica pela baia, movimentos repetidos de cabeça, dança de lobo ou síndrome do urso ou "weaving", aerofagia, morder madeira, coprofagia e lambedura de cocho).

Adicionalmente, para análise do comportamento, foi acessado o temperamento dos equinos por meio de entrevista com o tratador, sendo preenchido um questionário. Nesse questionário, a intensidade 
do temperamento foi classificada nos graus 1 a 3 , sendo o grau 1 de menor intensidade e o grau 3 de maior intensidade. Os temperamentos sugeridos foram brincalhão, curioso, nervoso e assustado (Leal 2007).

Com objetivo de verificar o ritmo circadiano do cortisol, colheram-se duas amostras de sangue de cada um dos animais, às 8:00h e às 17:00h. Após desinfecção do local com álcool iodado, foi feita a punção da jugular utilizando-se agulhas descartáveis e frascos dotados de sistema a vácuo. Cada colheita foi realizada com o animal em seu próprio ambiente, com a ajuda do tratador ou pessoa de contato próximo do animal, e em tempo inferior a 30 segundos, minimizando a ocorrência de estresse agudo ao mesmo. As amostras de sangue foram então centrifugadas e o soro separado e congelado à temperatura de $-20^{\circ} \mathrm{C}$. Para análise do cortisol sorológico foi utilizada a técnica de radioimunoensaio (RIE) de acordo com o protocolo do laboratório conveniado (Bet Laboratórios).

Foi utilizado o método proposto por Douglas (1999), (valor maior-menor)/valor maior, para definir a taxa de RCC de cada animal. Se o RCC fosse inferior a 0,30, considerou-se que o RCC esteja alterado. Os dados foram tabulados, obtendo-se as médias e os desvios padrões para os dados paramétricos e as frequências absolutas e percentuais para as ocorrências dos diversos tipos de comportamentos, temperamentos, cólicas e RCC alterados. Os escores para temperamento foram comparados entre si pelo teste de Kruskal-Wallis e as prevalências de escores com intensidade máxima (3) pelo testo de Qui-quadrado. Possíveis correlações entre escores de temperamento ou presença de comportamentos anormais com concentrações séricas de cortisol, taxa de RCC e frequências cardíaca e respiratória foram acessadas pelo teste de Spearman. Foi realizado teste exato de Fisher para comparar as incidências de RCC alterado dentro dos grupos de animais com e sem alteração comportamental. Para todos os testes considerou-se $\mathrm{P} \leq 0,05$.

\section{RESULTADOS E DISCUSSÃO}

Os parâmetros clínicos dos animais estudados mantiveram-se dentro dos padrões fisiológicos para a espécie (Quadro 1), com escores corporais variando entre 6 e 8 e baixos níveis de escoriações. Segundo a escala de Henneke et al. (1984), os animais apresentaram, portanto, escore entre pouco acima do peso e obeso. Atualmente a obesidade em equinos é preocupante pelas suas relações com pior performance atlética, termorregulação deficiente, risco de desenvolvimento de resistência à insulina e laminite (Quinn et al. 2006). Webb et al. (1990) mostraram que animais com escore corporal médio de 7,5 apresentavam pior resposta adaptativa ao exercício que animais com escore médio de 5,2.
Não foram relatadas cólicas no período de um ano anterior ao período de avaliação deste estudo. Estudos nacionais anteriores registraram altas incidências de cólica em animais de policiamento no Rio de Janeiro (Laranjeira et al. 2009), em animais do exército em Brasília (Vieira 2006) e em animais de policiamento estabulados em Belo Horizonte, também correlacionado a altas taxas de cortisol e comportamentos anormais (Leal 2007, Leal et al. 2011).

Questionários com tratadores, proprietários e adestradores são frequentemente utilizados como forma de avaliação do temperamento equino (Momozawa et al. 2005, Duberstein \& Gilkeson 2010, Suwała et al. 2016). No presente estudo não houve diferença estatística entre os graus de temperamento nervoso e assustado quando comparados entre si ou com os comportamentos curioso e brincalhão (Quadro 2).

A personalidade e o temperamento dos equinos são influenciados por diversos fatores. Sabe-se que existe um fator genético envolvido na personalidade (raça, linhagem) e que traços do temperamento são relacionados a experiências sofridas ao longo da vida (Hausberger et al. 2008, Suwała et al. 2016). A avaliação do temperamento do cavalo é considerada importante por proprietários e treinadores durante a compra, seja para fins de esporte ou lazer (Suwała et al. 2016). Entretanto tal avaliação por meio de questionários possui limitantes relativos a diferenças nas definições para certas características ou sentimentos por parte dos avaliadores e humanização do comportamento do animal, tornando necessários métodos objetivos de avaliação que corroborem com os achados (Hausberger et al. 2008). Estudos anteriores sugerem que existe relação entre temperamento e cortisolemia. Leal (2007) mostrou correlação positiva entre os temperamentos nervoso e assustado e altas taxas de cortisol sanguíneo em animais de policiamento de Belo Horizonte. Ellis et al. (2014) relataram maiores níveis de cortisol salivar matinal em cavalos classificados pelos cavalariços como excitados comparados aos classificados como calmos. No presente estudo, no entanto, não houve correlação significativa entre nenhum dos temperamentos, níveis de cortisol pela manhã ou tarde, taxa de RCC ou comportamentos anormais. Resguardadas as limitações da subjetividade da análise de comportamento, os elevados graus de estado geral e condição corporal, associados ao baixo grau de escoriações e da ausência de correlações entre comportamento e alterações hormonais indicam uma relação positiva e equilibrada entre tratadores e equinos participantes no presente estudo.

Quadro 1. Médias e desvios-padrão dos graus observados para estado geral, condição corporal e presença de escoriações e dos valores das frequências cardíaca (FC) e respiratória (FR) em equinos PSI Jockey Club do Paraná

\begin{tabular}{|c|c|c|c|c|c|}
\hline & Estado geral & Condição corporal & Escoriações & $\mathrm{FC}$ & FR \\
\hline & $(1-5)$ & $(1-9)$ & $(0-4)$ & (bat/min) & $(\mathrm{mov} / \mathrm{min})$ \\
\hline Média & 4,87 & 7,13 & 0,17 & 36,87 & 15,47 \\
\hline DP & 0,43 & 0,78 & 0,75 & 4,66 & 3,09 \\
\hline
\end{tabular}

Quadro 2. Médias e desvios padrões dos graus de temperamento observados em equinos do Jockey Club do Paraná

\begin{tabular}{|c|c|c|c|c|}
\hline & Brincalhão & Curioso & Nervoso & Assustado \\
\hline & $(1-3)$ & $(1-3)$ & $(1-3)$ & $(1-3)$ \\
\hline Média & 1,71 & 1,79 & 1,82 & 2 \\
\hline DP & 0,9 & 0,88 & 0,9 & 0,9 \\
\hline
\end{tabular}


Dos 30 animais observados, 11 apresentaram algum comportamento anormal durante o período de observação, sendo que um deles executou movimento de cavar e também movimentos aleatórios (Quadro 3). Três animais apresentaram comportamentos estereotipados orais (aerofagia, coprofagia e morder madeira).

Além do estresse (Mason \& Latham 2004) fatores ligados à nutrição (Johnson et al. 1998) e fase da carreira atlética (Mills et al. 2002) podem estar associados ao desenvolvimento de estereotipias. Segundo Johnson et al. (1998), alterações comportamentais são mais comuns em equinos alimentados com concentrado do que em animais alimentados somente com feno, provavelmente devido à redução do $\mathrm{pH}$ no intestino grosso dos primeiros. Todos os animais do estudo recebiam alimento concentrado pelo menos duas vezes ao dia e poderiam estar sob acidose intestinal. Segundo Mills et al. (2002), em cavalos Puro-Sangue Inglês o desenvolvimento de estereotipias pode estar relacionado a fatores estressantes como o início do treinamento (onde o bridão e a sela são impostas ao animal independente de sua reação ao equipamento), castração e o final da carreira de corridas (fêmeas que passam a ser usadas como matrizes). Neste estudo, os cavalos estavam em fase de treinamento ativo, não sofrendo estes efeitos do início ou fim da carreira.

Um percentual de $43,33 \%$ de todos os animais avaliados no estudo apresentaram RCC alterado. Sendo que cerca de dois terços desses $(63,64 \%)$ apresentaram alguma anomalia comportamental. (Quadro 4). Apesar de diferença estatística não ter sido detectada comparando-se esses grupos, tal como descrito em estudos prévios com equinos (Leal 2007, Leal et al. 2011), ressalta-se que a incidência de estereotipias foi duas vezes superior no grupo com alteração de RCC.

Dois animais deste estudo com RCC alterado também apresentaram agressividade como alteração comportamental e eram do sexo feminino. Segundo Douglas (1999), 85\% das éguas ou potras que apresentam RCC inferior a 30\% são criadas estabuladas e apresentam comportamento agressivo, o que pode estar relacionado ao estresse crônico.

Três animais estudados realizavam movimentos aleatórios na baia e eram impedidos de realizar plenamente a estereotipia por meio de "peias" (seus membros anteriores tiveram seus movimentos restringidos, presos um ao outro na altura da quartela). Um animal realizava coprofagia e por isso foi colocada uma "biqueira" (espécie de focinheira) para impedir este comportamento. Esses quatro animais, submetidos a métodos físicos para restringir a realização de comportamentos anormais, apresentaram alteração de RCC. Ao se comparar a incidência de RCC alterados nesse grupo com o grupo de animais que não apresentaram estereotipias verificou-se diferença estatística $(\mathrm{P}=0,024)$. Assim ao se aplicar o teste de odds ratio, verificou-se que animais submetidos a métodos físicos para restrição de estereotipias apresentam 18,7 (IC 0,87-402,1) vezes mais chance de apresentarem RCC alterado quando comparados com animais normais. Sabe-se que a realização de estereotipias pode estar relacionada a situações de adaptação encontrada pelo indivíduo para lidar com o ambiente. Por isso é considerado deletério impedir de formas física ou química a realização do comportamento anormal (Mason \& Latham 2004).

Segundo Mason \& Latham (2004), as estereotipias podem ser reflexos de quatro processos: 1 enriquecimento "faça você mesmo": o animal realiza o comportamento anormal em substituição a um comportamento natural (bovinos alimentados com concentrado que apresentam estereotipias orais como substituição à ruminação ou pastejo possuem menores níveis de hormônio adrenocorticotrófico, por exemplo); 2 efeito "mantra": movimentos repetitivos e rítmicos têm efeito calmante (comportamento observado em autistas e esquizofrênicos humanos, mas não comprovado em animais); 3 controle central: o comportamento efetuado

Quadro 3. Incidência de comportamentos normais e anormais observados em equinos PSI do Jockey Club do Paraná em 2009

\begin{tabular}{|c|c|c|c|c|c|}
\hline \multicolumn{3}{|c|}{ Normais } & \multicolumn{3}{|c|}{ Anormais } \\
\hline Tipo & Absoluta & Relativa & Tipo & Absoluta & Relativa \\
\hline Alerta & 15 & $50 \%$ & Aerofagia & 1 & $3,3 \%$ \\
\hline Deitado & 0 & $0 \%$ & Agressividade & 2 & $6,7 \%$ \\
\hline Distraído & 13 & $43,3 \%$ & Cavar & 1 & $3,3 \%$ \\
\hline Focinho chão & 4 & $13,3 \%$ & Coprofagia & 1 & $3,3 \%$ \\
\hline \multirow[t]{6}{*}{ Relinchar } & 1 & $3,3 \%$ & Dança lobo & 0 & $0 \%$ \\
\hline & & & Lamber cochos & 0 & $0 \%$ \\
\hline & & & Morder madeira & 1 & $3,3 \%$ \\
\hline & & & Mov. aleatórios & 6 & $20 \%$ \\
\hline & & & Mov. rep. cabeça & 0 & $0 \%$ \\
\hline & & & Geral & 11 & $36,7 \%$ \\
\hline
\end{tabular}

Quadro 4. Média \pm desvio padrão dos níveis de cortisol sérico matutino e vespertino e da taxa do ritmo circadiano de cortisol (RCC) dos equinos com alteração de RCC de acordo com o comportamento apresentado e médias gerais de todos os animais do Jockey Club do Paraná

\begin{tabular}{|c|c|c|c|c|}
\hline & $\begin{array}{l}\text { Incidência de animais } \\
\text { com RCC alterado }\end{array}$ & $\begin{array}{l}\text { Níveis de cortisol }(\mathrm{ng} / \mathrm{ml}) \\
\text { da manhã }\end{array}$ & $\begin{array}{l}\text { Níveis de cortisol }(\mathrm{ng} / \mathrm{ml}) \\
\text { da tarde }\end{array}$ & Taxa RCC \\
\hline Animais com comportamento anormal & $63,64 \%$ & $65,87 \pm 7,70$ & $65,49 \pm 12,57$ & $0,16 \pm 0,10$ \\
\hline Animais com comportamento normal & $31,58 \%$ & $64,12 \pm 13,33$ & $58,85 \pm 18,1$ & $0,19 \pm 0,07$ \\
\hline Média geral $(n=30)$ & $43,33 \%$ & $73,61 \pm 31,27$ & $44,62 \pm 27,12$ & $0,44 \pm 0,27$ \\
\hline
\end{tabular}


repetidamente passa a ser realizado automaticamente, com mínimo processamento cognitivo e independente da continuidade do estímulo; 4 comportamento perseverante: repetição obsessiva do comportamento mesmo sem repetição do estímulo. Os resultados do presente estudo corroboram as observações desses autores de que as estereotipias nem sempre devem ser combatidas, podem demorar ou jamais deixarem de ocorrer em alguns animais e, isoladamente, são um indicador pobre e controverso do bem-estar animal, ressaltando a importância da associação de outros parâmetros indicadores de bem-estar.

A realização de estereotipias pode reduzir os sinais fisiológicos de estresse, sendo considerada uma forma de o animal superar o estresse e se adaptar (Cooper \& Nicol 1993, Mason \& Latham 2004). Isso sugere, portanto, que os animais que apresentaram comportamento anormal e taxa de RCC normal se adaptaram à condição estressante imposta a eles por meio das estereotipias, enquanto os animais com comportamento normal e RCC alterado não foram capazes de lidar com o estresse de forma satisfatória, refletindo na sua função adrenocortical. Outra hipótese, além da adaptação, levantada por Irvine \& Alexander (1994), é a de que cavalos de corrida apresentam um ciclo circadiano de cortisol normal mesmo considerando-se a rotina artificial à qual são submetidos devido às suas generosas refeições com concentrado e exercícios, responsáveis por aumentar os níveis de cortisol durante o dia. De acordo com essa premissa, haveria o risco de alguns animais com RCC alterado não serem identificados devido à influência da dieta e atividades sobre a cortisolemia. No entanto, neste trabalho, as coletas de sangue matinais foram realizadas antes da alimentação e dos exercícios, impedindo esta interferência nos resultados encontrados.

\section{CONCLUSÕES}

A população de equinos do Jockey Club do Paraná avaliada no presente estudo apresentou parâmetros clínicos condizentes com um bom estado de saúde física e ausência de cólica nos doze meses anteriores, o que demonstra bem-estar adequado quanto às necessidades físicas e nutricionais. A mínima ocorrência de escoriações indica boas práticas dos tratadores no manejo dos equinos.

Contudo, a elevada prevalência de comportamentos anormais e alterações do ritmo circadiano de cortisol, principalmente nos animais submetidos a métodos para restrição da manifestação de estereotipia, demonstram um quadro de estresse crônico e inadequação do bem-estar psíquico.

Novos estudos deverão ser realizados para comprovar a associação entre prevalência de alterações no RCC e no comportamento e objetivando buscar alternativas terapêuticas e de manejo para a melhoria dessas condições.

\section{REFERÊNCIAS}

Barnett J.L. \& Hemsworth P.H. 1990. The validity of physiological and behavioural measures of animal welfare. Appl. Anim. Behav. Sci. 25(1/2):177-187. <http://dx.doi.org/10.1016/0168-1591(90)90079-S>

Broom D.M. \& Molento C.F.M. 2004. Bem-estar animal: conceito e questões relacionadas, revisão. Arch. Vet. Sci. 9(2):1-11.<http://dx.doi.org/10.5380/ avs.v9i2.4057>

Budzyńska M. 2014. Stress reactivity and coping in horse adaptation to environment. J. Equine Vet. Sci. 34(8):935-941. <http://dx.doi.org/10.1016/j. jevs.2014.05.010>
Carroll C.L. \& Huntington P.J. 1988. Body condition scoring and weight estimation of horses. Equine Vet. J. 20(1):41-45. <http://dx.doi. org/10.1111/j.2042-3306.1988.tb01451.x><PMid:3366105>

Cooper J.J. \& Nicol C.J. 1993. The 'coping' hypothesis of stereotypic behaviour: a reply to rushen. Anim. Behav. 45(3):616-618. <http://dx.doi.org/10.1006/ anbe.1993.1072>

Douglas R. 1999. Circadian cortisol rhythmicity and equine Cushing's-like disease. J. Equine Vet. Sci. 19(11):684-753. <http://dx.doi.org/10.1016/ S0737-0806(99)80111-7>

Duberstein K.J. \& Gilkeson J.A. 2010. determination of sex differences in personality and trainability of yearling horses utilizing a handler questionnaire. Appl. Anim. Behav. Sci. 128(1):57-63. <http://dx.doi. org/10.1016/j.applanim.2010.09.012>

Ellis A.D., Stephenson M., Preece M. \& Harris P. 2014. a novel approach to systematically compare behavioural patterns between and within groups of horses. Appl. Anim. Behav. Sci. 161:60-74. <http://dx.doi.org/10.1016/j. applanim.2014.09.017>

Gontijo L.D.A., Cassou F., Michelotto Junior P.V., Alves G.E.S., Bringel B., Ribeiro R.M., Lago L.A. \& Faleiros R.R. 2014. Bem-estar em equinos de policiamento em Curitiba/PR: indicadores clínicos, etológicos e ritmo circadiano do cortisol. Ciência Rural 44(7):1272-1276. <http://dx.doi. org/10.1590/0103-8478cr20131196>

Hausberger M., Roche H., Henry S. \& Visser E.K. 2008. A review of the humanhorse relationship. Appl. Anim. Behav. Sci. 109(1):1-24. <http://dx.doi. org/10.1016/j.applanim.2007.04.015>

Henneke D.R., Potter G. \& Kreider J.L. 1984. Body condition during pregnancy and lactation and reproductive efficiency of mares. Theriogenology 21(6):897-909. <http://dx.doi.org/10.1016/0093-691X(84)90383-2>

Irvine C.H.G. \& Alexander S.L. 1994. Factors affecting the circadian rhythm in plasma cortisol concentrations in the horse. Domest. Anim. Endocrinol. 11(2):227-238. <http://dx.doi.org/10.1016/0739-7240(94)90030-2> $<$ PMid:8045104>

Johnson K.G., Tyrrell J., Rowe J.B. \& Pethick D.W. 1998. Behavioural changes in stabled horses given nontherapeutic levels of virginiamycin. Equine Vet. J. 30(2):139-143. <http://dx.doi.org/10.1111/j.2042-3306.1998. tb04473.x><PMid:9535070>

Laranjeira P.V.E.H., Almeida F.Q.D., Pereira J.S., Lopes M.A.F.L., Campos C.H.C.D., Caiuby L.C.A.B. \& Souza P.N.B.D. 2009. Perfil e distribuição da síndrome cólica em equinos em três unidades militares do estado do Rio de Janeiro, Brasil. Ciência Rural 39(4):1108-1115. <http://dx.doi.org/10.1590/ S0103-84782009005000043>

Leal B.B. 2007. Avaliação do bem-estar dos equinos de cavalaria da Polícia Militar de Minas Gerais: indicadores Etológicos, Endocrinológicos e incidência de cólica. Dissertação de Mestrado em Medicina Veterinária, Escola de Veterinária, Universidade Federal de Minas Gerais, Belo Horizonte. 61p.

Leal B.B., Alves G.E., Douglas R.H., Bringel B., Young R.J., Haddad J.P.A., Viana W.S. \& Faleiros R.R. 2011. Cortisol circadian rhythm ratio: a simple method to detect stressed horses at higher risk of colic? J. Equine Vet. Sci. 31(4):188190. <http://dx.doi.org/10.1016/j.jevs.2011.02.005>

Mason G.J. \& Latham N.R. 2004. Can't stop, won't stop: is stereotypy a reliable animal welfare indicator? Anim. Welf. 13:S57-S70.

Mills D.S., Alston R.D., Rogers V. \& Longford N. 2002. Factors associated with the prevalence of stereotypic behaviour amongst thoroughbred horses passing through auctioneer sales. Appl. Anim. Behav. Sci. 78(2):115-124. <http://dx.doi.org/10.1016/S0168-1591(02)00096-5>

Momozawa Y., Kusunose R., Kikusui T., Takeuchi Y. \& Mori Y. 2005. Assessment of equine temperament questionnaire by comparing factor structure between two separate surveys. Appl. Anim. Behav. Sci. 92(1):77-84.<http://dx.doi. org/10.1016/j.applanim.2004.11.006> 
Quinn R.W., Burk A.O., Suagee J.K. \& Hartsock T.G. 2006. Equine obesity: implications for health and strategies for control. Proceedings of The 4th Mid-Atlantic Nutrition Conference, Timonium, Maryland, p.23-36. (Resumo)

Rushen J. 1991. Problems associated with the interpretation of physiological data in the assessment of animal welfare. Appl. Anim. Behav. Sci. 28(4):381386. <http://dx.doi.org/10.1016/0168-1591(91)90170-3>

Søndergaard E. \& Ladewig J. 2004. Group housing exerts a positive effect on the behaviour of young horses during training. Appl. Anim. Behav. Sci. 87(1):105-118. <http://dx.doi.org/10.1016/j.applanim.2003.12.010>

Suwała M., Górecka-Bruzda A., Walczak M., Ensminger J. \& Jezierski T. 2016. A desired profile of horse personality a survey study of polish equestrians based on a new approach to equine temperament and character. Appl. Anim. Behav.Sci. 180:65-77.<http://dx.doi.org/10.1016/j.applanim.2016.04.011>

Vieira A.R.A. 2006. Prevalência e desgaste anormal dos dentes incisivos e incidência de cólica em equinos estabulados apresentando distúrbios de comportamento: análise de um total de 407 equinos. Dissertação de Mestrado em Medicina Veterinária, Escola de Veterinária, Universidade Federal de Viçosa, Viçosa. 47p.

Webb S.P., Potter G.D., Evans J.W. \& Webb G.W. 1990. Influence of body fat content on digestible energy requirements of exercising horses in temperate and hot environments. J. Equine Vet. Sci. 10(2):116-120. <http://dx.doi. org/10.1016/S0737-0806(06)80118-8> 